University of Nebraska - Lincoln

DigitalCommons@University of Nebraska - Lincoln

$1-26-2005$

\title{
Circularly polarized laser field-induced rescattering plateaus in electron-atom scattering
}

A. V. Flegel

Voronezh State University, Voronezh 394006 , Russia

M. V. Frolov

Voronezh State University, Voronezh, Russia, and University of Nebraska - Lincoln

N. L. Manakov

Voronezh State University, manakov@phys.vsu.ru

Anthony F. Starace

University of Nebraska-Lincoln, astarace1@unl.edu

Follow this and additional works at: https://digitalcommons.unl.edu/physicsstarace

Part of the Physics Commons

Flegel, A. V.; Frolov, M. V.; Manakov, N. L.; and Starace, Anthony F., "Circularly polarized laser field-induced rescattering plateaus in electron-atom scattering" (2005). Anthony F. Starace Publications. 111.

https://digitalcommons.unl.edu/physicsstarace/111

This Article is brought to you for free and open access by the Research Papers in Physics and Astronomy at DigitalCommons@University of Nebraska - Lincoln. It has been accepted for inclusion in Anthony F. Starace Publications by an authorized administrator of DigitalCommons@University of Nebraska - Lincoln. 


\title{
Circularly polarized laser field-induced rescattering plateaus in electron-atom scattering
}

\author{
A.V. Flegel ${ }^{a}$, M.V. Frolov ${ }^{a, b}$, N.L. Manakov ${ }^{a}$, Anthony F. Starace $^{b}$ \\ a Department of Physics, Voronezh State University, Voronezh 394006, Russia \\ ${ }^{b}$ Department of Physics and Astronomy, The University of Nebraska, Lincoln, NE \\ 68588-0111, USA
}

\begin{abstract}
We analyze the laser ellipticity dependence of $n$-photon differential cross sections $\left(\mathrm{d} \sigma_{n} / \mathrm{d} \Omega\right)$ for electron-atom scattering in an intense elliptically polarized laser field. We show that there exist two plateau-like structures in the dependence of $\mathrm{d} \sigma_{n} / \mathrm{d} \Omega$ on $n$ for any ellipticity, including for the case of circular polarization. We present numerical predictions for e- $\mathrm{H}$ scattering in a $\mathrm{CO}_{2}$-laser field and an analytical description of the plateau features in terms of the rescattering scenario.
\end{abstract}

Key words: Electron-atom scattering; Strong laser field; Rescattering effects; Circular dichroism

PACS: 03.65.Nk, 34.80.Qb, 34.50.Rk, 32.80.Wr

\section{Introduction}

Plateau structures in intense laser-atom interactions (i.e., a nearly constant dependence of multiphoton cross sections on photon number $n$ over a wide interval of $n$ up to a cutoff at $n_{\max }$ ) are among the most interesting and intensively studied nonlinear phenomena in laser-atom physics. These structures have a one-electron origin and are well-studied both experimentally and theoretically for the processes of above-threshold ionization (ATI) and high harmonic generation (HHG) [1]. Recently, plateau structures have been predicted also for the process of laser-assisted electron-atom scattering (LAES) [2]. Detailed theoretical analyses of plateau effects have been performed for the case of linear laser polarization, for which a one-dimensional model of electron motion along the direction of laser polarization is applicable and for which a numerical analysis of the time-dependent Schrödinger equation is simplified owing to the conservation of the electron's angular momentum projection 
along the direction of laser polarization. The rescattering picture [3] provides a transparent physical explanation for the appearance of plateau structures: an intense oscillating laser field returns ionized electrons back to the parent ion, whereupon they either gain additional energy from the laser field during laser-assisted collisional events, thereby forming the high-energy plateau in the ATI spectrum, or recombine with the parent ion, emitting high-order harmonic photons. A similar interpretation of the high-energy plateau for LAES as well as analytical estimates for $n_{\max }$ for the case of a linearly polarized field are given in Ref. [2].

For the case of an elliptically polarized laser field, rescattering effects in LAES spectra have not yet been analyzed. The high-energy (rescattering) plateaus in ATI and HHG spectra, however, are known to gradually disappear with increasing degree of circular polarization $|\xi|(-1 \leq \xi \leq+1)$ [1]. Indeed, for the case of pure circular polarization $(\xi= \pm 1)$, the process of HHG by free atoms is strictly forbidden, whereas plateau structures in ATI simply disappear. Thus it has been generally assumed that rescattering effects vanish for the case of circular polarization owing to the impossibility for the electron to return to its parent ion. However, for free-free transitions (such as LAES), rescattering effects can take place even for the case of circular polarization, as follows from quite general arguments. For the case of bound-bound or boundfree transitions (i.e., HHG or ATI), the angular momentum $l$ of the bound electron (having energy $E_{0}$ ) is fixed, so that dipole selection rules for the angular momentum projection $m$ in a circularly polarized field, $|\Delta m|=1$, forbid HHG and suppress rescattering effects in ATI. (The suppression of ATI occurs because after absorption of $n>n_{0} \approx\left(\left|E_{0}\right| / \hbar \omega\right)$ photons, the ionized electron acquires a large additional angular momentum, $\Delta l=n$, whose centrifugal potential barrier makes recollision improbable.) For the case of LAES, however, both incoming and scattered electron waves are superpositions of continuum states with different $l$ and $m$. Hence, for LAES the selection rules should not lead to such drastic differences in the physics of strong field phenomena for the cases of linear and circular polarizations as they do for ATI and HHG.

The main purpose of this Letter is to demonstrate the existence of rescattering effects (and corresponding plateau features) for free-free electron transitions in the presence of a circularly polarized laser field. In contrast to ATI and HHG, where the height of plateau structures decreases rapidly with increasing ellipticity, we find that for the case of LAES the plateau height is almost insensitive to the degree of circular polarization $\xi$, whose magnitude and sign determine only the extent of the high-energy plateau region. These features of plateau structures in LAES are shown to follow from an exact quantum solution of the problem, which allows also for a simple classical interpretation in terms of the rescattering picture. 


\section{Formulation of the problem and basic equations}

Theoretical analysis of LAES from a neutral atom is simpler than the analysis of either ATI or HHG since the atomic potential $U(r)$ does not involve a long-range Coulomb tail. Therefore, for slow incident electrons (the case for which rescattering effects are most important) the electron-atom interaction can be modelled by a zero-range potential (ZRP), which supports a weaklybound $s$-state having energy $E_{0}=-\hbar^{2} \kappa^{2}(2 m)^{-1}$. For LAES, this approximation represents a time-dependent extension of the standard scattering length approximation for the description of low-energy, $s$-wave electron scattering from atoms that have negative ions with $s$-electron ground states [4]. ${ }^{1}$

In the dipole approximation, we describe the laser field by the electric vector,

$$
\mathbf{F}(t)=F \operatorname{Re}\{\mathbf{e} \exp (-\mathrm{i} \omega t)\}, \quad \mathbf{e}=(\hat{\boldsymbol{\varepsilon}}+\mathrm{i} \eta[\hat{\mathbf{k}} \times \hat{\varepsilon}]) / \sqrt{1+\eta^{2}}
$$

where $F, \omega$ and $\mathbf{e}$ are the amplitude, frequency and complex polarization vector, $\left(\mathbf{e} \cdot \mathbf{e}^{*}=1\right)$. The unit vectors $\hat{\varepsilon}$ and $\hat{\mathbf{k}}$ define the major semiaxis of the laser polarization ellipse and the direction of the laser beam. Instead of the ellipticity $\eta$, it is convenient to use the degrees of linear $(\ell)$ and circular $(\xi)$ polarization: $\ell=\mathbf{e} \cdot \mathbf{e}=\left(1-\eta^{2}\right) /\left(1+\eta^{2}\right), \xi=\mathbf{i} \hat{\mathbf{k}} \cdot\left[\mathbf{e} \times \mathbf{e}^{*}\right]=2 \eta /\left(1+\eta^{2}\right)$. Since $\left|E_{0}\right|$ (or the scattering length $\kappa^{-1}$ ) is the only free parameter of the problem, we use scaled units in which electron energies and $\hbar \omega$ are measured in units of $\left|E_{0}\right|$, momenta in units of $\hbar \kappa=\sqrt{2 m\left|E_{0}\right|}$, the field amplitude in units of $F_{0}=\sqrt{2 m\left|E_{0}\right|^{3}} /(e \hbar)$, and cross sections in units of $\kappa^{-2}$. As an example, for $e-H$ scattering, $\left|E_{0}\right|=0.754 \mathrm{eV}=0.0277$ a.u. is the binding energy of the $\mathrm{H}^{-}$ion and $F_{0}=3.36 \times 10^{7} \mathrm{~V} / \mathrm{cm}=6.52 \times 10^{-3}$ a.u.; the scaled unit for the laser intensity, $I=c F^{2} /(8 \pi)$, is thus $I_{0}=1.5 \times 10^{12} \mathrm{~W} / \mathrm{cm}^{2}=4.3 \times 10^{-5}$ a.u.

A periodic function, $f_{\mathbf{p}}(t)=\sum_{k} f_{k} \exp (-\mathrm{i} k \omega t)$, plays a key role in the description of LAES for a ZRP model. Namely, it determines the behavior at the origin of the exact scattering state, $\Phi_{\mathbf{p}}(\mathbf{r}, t)$, for an incident electron having momentum $\mathbf{p}$ and energy $E=p^{2}$ :

$$
\left.\Phi_{\mathbf{p}}(\mathbf{r}, t)\right|_{\mathbf{r} \rightarrow 0}=\left(r^{-1}-1\right) f_{\mathbf{p}}(t) .
$$

(For the explicit form of $\Phi_{\mathbf{p}}(\mathbf{r}, t)$ see Ref. [2].) The function $f_{\mathbf{p}}(t)$ contains the complete dynamical information of the electron-atom interaction in the

$\overline{1}$ Note that the effective range theory [4] may be used to provide a more precise account of $U(r)$ by introducing the effective range, $r_{0}$, as well as the scattering length $\kappa^{-1}$. (ATI in this approach has been considered in [5].) However, we have found that the present results for LAES are not changed qualitatively by introducing $r_{0}$. 
presence of a strong laser field. Its Fourier-coefficients $f_{k}$ determine the exact amplitude, $\mathcal{A}_{n}$, for multichannel electron scattering with absorption $(n>0)$ or emission $(n<0)$ of $|n|$ photons. The infinite system of linear algebraic equations for the coefficients $f_{k}$, obtained in Ref. [2], is equivalent to the following inhomogeneous integral equation for $f_{\mathbf{p}}(t)$ :

$$
\begin{aligned}
& (1+\mathrm{i} p) f_{\mathbf{p}}(t)+c_{\mathbf{p}}(t)=-\frac{1}{\sqrt{4 \pi i}} \int_{0}^{\infty} \frac{\mathrm{d} \tau}{\tau^{3 / 2}} \mathrm{e}^{\mathrm{i} E \tau} \\
& \times\left(f_{\mathbf{p}}(t-\tau) \mathrm{e}^{\mathrm{i} u_{p} \tau+\mathrm{i} S(t, t-\tau)}-f_{\mathbf{p}}(t)\right),
\end{aligned}
$$

where $c_{\mathbf{p}}(t)$ is the time-dependent part of the quasienergy wave function, $\Phi_{\mathbf{p}}^{(0)}(\mathbf{r}, t)$, of a free electron in the field $\mathbf{F}(t)$ :

$$
\begin{aligned}
& c_{\mathbf{p}}(t)=\Phi_{\mathbf{p}}^{(0)}(\mathbf{r}=0, t) \\
& =\exp \left[\mathrm{i}\left(E+u_{p}\right) t-\mathrm{i} \int^{t}\left(\mathbf{p}+\mathbf{A}\left(t^{\prime}\right)\right)^{2} \mathrm{~d} t^{\prime}\right],
\end{aligned}
$$

where $\mathbf{A}(t)=\dot{\mathbf{F}}(t) / \omega^{2}$ is the vector potential and $u_{p}=F^{2} /\left(2 \omega^{2}\right)$ is the ponderomotive shift $\left(u_{p}=e^{2} F^{2} /\left(4 m \omega^{2}\right)\right.$ in abs. units). In Eq. (1), $S(t, t-\tau) \equiv$ $S\left(\mathbf{r}=0, t ; \mathbf{r}^{\prime}=0, t-\tau\right)$ is the classical action for a free electron in the laser field:

$$
S(t, t-\tau)=-\int_{t-\tau}^{t} \mathbf{A}\left(t^{\prime}\right)^{2} \mathrm{~d} t^{\prime}+\frac{1}{\tau}\left(\int_{t-\tau}^{t} \mathbf{A}\left(t^{\prime}\right) d t^{\prime}\right)^{2} .
$$

The exact result for the amplitude $\mathcal{A}_{n}$ in terms of $f_{k}$ has been obtained in [2] and may be rewritten as follows:

$$
\begin{aligned}
\mathcal{A}_{n} & =\sum_{k=-\infty}^{\infty} \mathrm{i}^{k} f_{n-k} \sum_{s=-\infty}^{\infty} \mathrm{e}^{\mathrm{i}(k+2 s) \arg \left(\mathbf{e} \cdot \mathbf{p}_{n}\right)} \\
& \times J_{k+2 s}\left(\frac{2 F}{\omega^{2}}\left|\mathbf{e} \cdot \mathbf{p}_{n}\right|\right) J_{s}\left(\ell \frac{u_{p}}{2 \omega}\right)
\end{aligned}
$$

where $\mathbf{p}_{n}$ is the scattered electron momentum $\left(p_{n}^{2}=E_{n}=E+n \omega\right)$ and $J_{m}(x)$ is a Bessel function. For analytical analyses of plateau structures in the differential cross sections $\mathrm{d} \sigma_{n} / \mathrm{d} \Omega=\left(p_{n} / p\right)\left|\mathcal{A}_{n}\right|^{2}$, it is convenient to express $\mathcal{A}_{n}$ as the following Fourier-integral (which reduces to (4) after expansion of $f_{\mathbf{p}}(t)$ and $c_{\mathbf{p}_{n}}^{*}(t)$ in Fourier series):

$$
\mathcal{A}_{n}=\frac{\omega}{2 \pi} \int_{0}^{2 \pi / \omega} c_{\mathbf{p}_{n}}^{*}(t) f_{\mathbf{p}}(t) \mathrm{e}^{\mathrm{i} n \omega t} \mathrm{~d} t .
$$



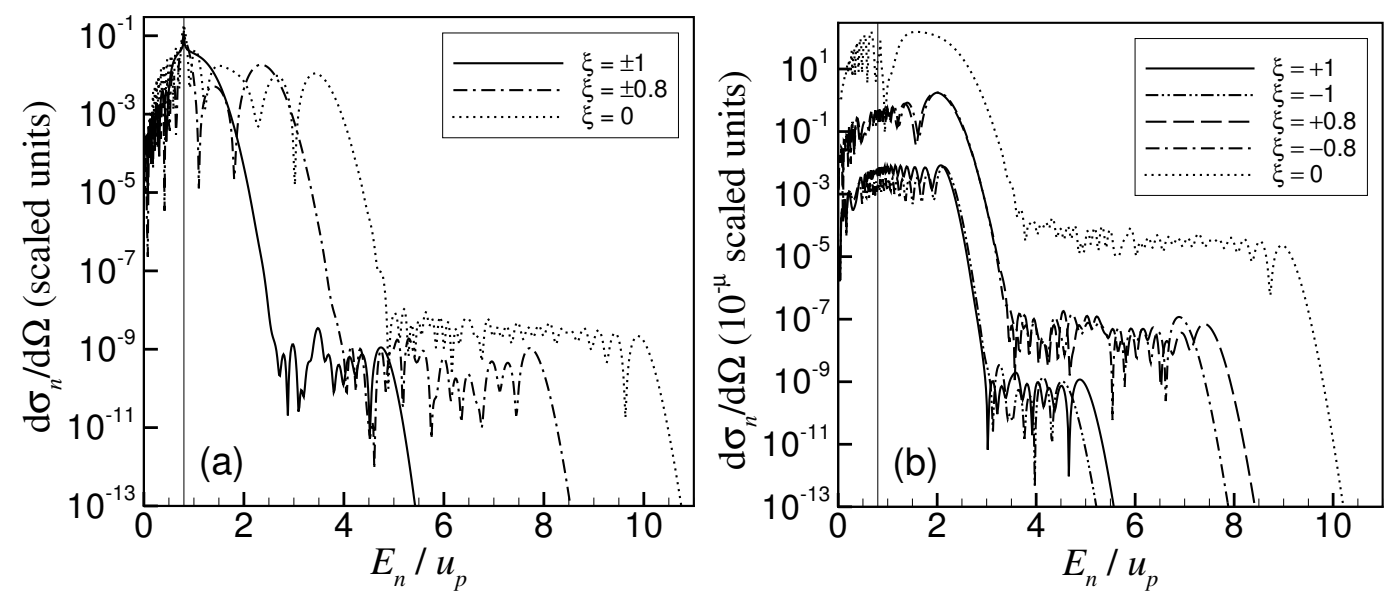

Fig. 1. (a) Spectra of LAES for forward scattering $\left(\mathbf{p}_{n} \| \mathbf{p}\right)$ along the major axis of the laser polarization ellipse. The incoming electron energy is $E=7.03=0.8 u_{p}$, and the laser parameters are $F=0.65, \omega=0.155$ and five different values of $\xi$, as indicated in the figure. The vertical line denotes elastic scattering, $E_{n}=E$. (b) Same as (a), but for the scattering angle $\theta=20^{\circ}$ in the plane of the polarization ellipse. For better visualization of the curves for different values of $\xi$, each one is multiplied by a factor $10^{\mu}$, where $\mu=4$ for $\xi=0, \mu=2$ for $\xi= \pm 0.8$ and $\mu=0$ for $\xi= \pm 1$.

Note that when the laser field is turned off (i.e., $F \rightarrow 0$ ), $c_{\mathbf{p}}(t) \rightarrow 1$ and one finds from Eq. (1) that $f_{k} \rightarrow f_{0} \delta_{k, 0}$. Consequently, both the function $f_{\mathbf{p}}(t)$ in Eq. (1) and the amplitude $\mathcal{A}_{0}$ in Eq. (5) (where $\mathcal{A}_{n} \rightarrow \mathcal{A}_{0} \delta_{n, 0}$ ) reduce to the amplitude $f_{0}(p)$ for low-energy ( $s$-wave) electron scattering from a short-range potential $U(r), f_{0}(p)=-(1+i p)^{-1}$ (cf. Eq.(133.7) in [4]).

\section{$3 \quad$ Numerical results for LAES spectra}

The present numerical results show that plateau structures in the $n$-dependence of LAES cross sections, $\mathrm{d} \sigma_{n} / \mathrm{d} \Omega=\left(p_{n} / p\right)\left|\mathcal{A}_{n}\right|^{2}$, appear in the presence of an intense low-frequency laser field (at $\omega<1$ and $u_{p} \gg \omega$ ) and that they are most pronounced when the initial momentum $\mathbf{p}$ is directed along the major polarization axis and the scattering angle $\theta$ is small (where $\theta$ is the angle between $\mathbf{p}_{n}$ and p). Fig. 1 presents $d \sigma_{n} / d \Omega$, calculated using the exact amplitude (4), as a function of the ratio $E_{n} / u_{p}$ for two values of $\theta$ and different values of the polarization parameter $\xi$. For the case of $e-H$ scattering, the scaled parameters $\omega, E$ and $F$ in Fig. 1 correspond to $\mathrm{CO}_{2}$-laser radiation $(\lambda=10.6 \mu \mathrm{m})$ of intensity $I=6.34 \times 10^{11} \mathrm{~W} / \mathrm{cm}^{2}$ and an initial electron energy $E=5.31 \mathrm{eV}$. Fig. 1 shows that two plateau-like structures in the energy distribution of scattered electrons with $E_{n}>E$, predicted in Ref. [2] for the case of linear polarization (i.e., $\xi=0, \ell=1$ ), exist also for an elliptically polarized field, including for the case of circular polarization $(\xi= \pm 1)$. More- 
over, for non-zero scattering angle $\theta$, an interesting polarization phenomenon (circular dichroism) appears: the electron angular distributions, including the high-energy plateau structures and their cutoffs, $E_{n, \max }$, depend upon the sign of $\xi$, i.e., upon the handedness of the laser photons. In addition, for $\xi \neq 0$, the extent of the high-energy plateau is maximal for $\theta \neq 0$. Dichroic effects for LAES, as well as for other atomic photoprocesses [6], have an interference origin, so that LAES experiments with an elliptically polarized laser field allow one to obtain more complete information about the details of LAES than is possible for the case of $\xi=0$.

\section{Analytical analysis of plateau structures in LAES}

To establish the physical origin of plateau structures in LAES spectra, we perform an analytical evaluation of the integral (5) for the amplitude $\mathcal{A}_{n}$ taking into account the zero order and first iteration of Eq. (1) for $f_{\mathbf{p}}(t)$, i.e.,

$$
\begin{aligned}
& f_{\mathbf{p}}(t) \approx f_{\mathbf{p}}^{(0)}(t)+f_{\mathbf{p}}^{(1)}(t) \\
& f_{\mathbf{p}}^{(0)}(t)=-\frac{c_{\mathbf{p}}(t)}{1+\mathrm{i} p}, \\
& f_{\mathbf{p}}^{(1)}(t)=\frac{1}{(1+\mathrm{i} p)^{2} \sqrt{4 \pi \mathrm{i}}} \\
& \times \int_{0}^{\infty} \frac{d \tau}{\tau^{3 / 2}}\left(e^{\mathrm{i}\left[\varphi(t, \tau)+\left(E+u_{p}\right) t\right]}-c_{\mathbf{p}}(t) e^{\mathrm{i} E \tau}\right), \\
& \varphi(t, \tau)=S(t, t-\tau)-\int^{t-\tau}\left(\mathbf{p}+\mathbf{A}\left(t^{\prime}\right)\right)^{2} \mathrm{~d} t^{\prime} .
\end{aligned}
$$

Numerical analysis shows that the approximation (6) reproduces accurately the results of the exact calculations for $\mathrm{d} \sigma_{n} / \mathrm{d} \Omega$ that are shown in Fig. 1. Moreover, the low-energy part of the electron spectrum (i.e., the $K$-plateau [2]) is found to be described by the amplitude $\mathcal{A}_{n}^{(0)}$ corresponding to the zero order function, $f_{\mathbf{p}}^{(0)}(t)$, whereas the high-energy $(R)$ plateau is found to originate from the amplitude $\mathcal{A}_{n}^{(1)}$ that corresponds to $f_{\mathbf{p}}^{(1)}(t){ }^{2}$

$\overline{2}$ As has been shown in Ref. [2], the approximation (7) is equivalent to the KrollWatson (low-frequency) approximation [7] and is similar to the Keldysh approximation [8] in the theory of tunnel ionization: both approximations correspond to a "minimal" account of the atomic potential effects and thus fail to describe the high-energy plateaus in LAES and ATI. 


\subsection{Low energy $(K)$ plateau}

Owing to the rapidly oscillating time-dependence of the functions $c_{\mathbf{p}}(t)$ and $c_{\mathbf{p}_{n}}^{*}(t)$ for small $\omega$, in order to estimate the position of the $K$-plateau cutoff, we evaluate the integral over $t$ in Eq. (5) with $f_{\mathbf{p}}(t) \approx f_{\mathbf{p}}^{(0)}(t)$ using the saddle point method. The saddle points, $t=t_{s}$, are given by the equation,

$$
\left(\mathbf{p}+\mathbf{A}\left(t_{s}\right)\right)^{2}=\left(\mathbf{p}_{n}+\mathbf{A}\left(t_{s}\right)\right)^{2},
$$

which allows one to interpret LAES as a single collisional event in which an incoming electron changes its momentum from $\mathbf{p}$ to $\mathbf{p}_{n}$ at the moment $t_{s}$ while conserving its mechanical kinetic energy (as expressed by the equality of the lhs and the rhs in Eq. (10)). Equation (10) has both real and complex roots $t_{s}$. In the former case the amplitude $\mathcal{A}_{n}^{(0)}$ oscillates as a function of $n$ ("classically-allowed scattering"), whereas in the latter case $\mathcal{A}_{n}^{(0)}$ decays exponentially. Thus, the cutoff of the $K$-plateau corresponds to the border between classically allowed and forbidden scattering, i.e., to the minimum value of $p_{n}$ at which the roots of Eq. (10) become complex. This estimate agrees well with the results of the exact calculations and for scattering in the plane of the polarization ellipse (as in Fig. 1) gives the following expression for the momentum $p_{n, \max }^{(0)}$ of the scattered electrons at the $K$-plateau cutoff:

$$
\begin{array}{r}
p_{n, \max }^{(0)}(\theta, \ell)=\sqrt{(1+\ell) u_{p}} \max \left(\sin \phi_{s}\right. \\
\left.+\sqrt{\left(\sin \phi_{s}-a\right)^{2}-4 a \sin (\tilde{\theta} / 2) \cos \left(\phi_{s}+\tilde{\theta} / 2\right)}\right),
\end{array}
$$

where $a=p / \sqrt{(1+\ell) u_{p}}, \tilde{\theta}=\arg \left(\mathbf{e} \cdot \mathbf{p}_{n}\right)-\arg (\mathbf{e} \cdot \mathbf{p})$ and the maximum is calculated over the set of values of the parameter $\phi_{s} \equiv \omega t_{s}, s=1,2, \ldots$ (i.e., over the phase of the laser field at the moment of electron-atom collision in accordance with Eq. (10)). Note that the expression that is maximized on the rhs of Eq. (11) is invariant to the substitution $\xi \rightarrow-\xi$, so that the position of the $K$-plateau cutoff does not depend on the sign of the photon helicity. For forward and back scattering $\left(\tilde{\theta}=\theta=0^{\circ}\right.$ and $\left.180^{\circ}\right)$, the maximum in Eq. (11) can be calculated analytically:

$$
p_{n, \max }^{(0)}=2 \sqrt{(1+\ell) u_{p}} \mp p, \quad p_{n}>p,
$$

where the signs $-/+$ correspond to forward/back scattering. Eq. (12) shows that the $K$-plateau for forward scattering exists only for incoming electron energies $E<(1+\ell) u_{p}$ and its extent decreases with increasing $E$ starting from the maximum value, $E_{n, \max }^{(0)}=4(1+\ell) u_{p}$, at $E \rightarrow 0$. 


\subsection{Basic equations for the rescattering scenario in LAES}

Rescattering effects are described by the amplitude $\mathcal{A}_{n}^{(1)}$. To evaluate it and to derive the equations that justify the rescattering scenario, we first extract the rapidly oscillating part from the function $f_{\mathbf{p}}^{(1)}(t)$ (see Eq. (16)) using the saddle point method to evaluate the integral over $\tau$ in Eq. (8) that involves the rapidly oscillating (over $t$ and $\tau$ ) function $\varphi(t, \tau)$. The equation for the saddle points $\tau_{s}=\tau_{s}(t), \partial \varphi(t, \tau) / \partial \tau=0$, is equivalent to the following relation:

$$
\left(\mathbf{p}+\mathbf{A}\left(t-\tau_{s}\right)\right)^{2}=\left(\mathbf{k}\left(t, \tau_{s}\right)+\mathbf{A}\left(t-\tau_{s}\right)\right)^{2},
$$

where we have introduced the "intermediate momentum" $\mathbf{k}$,

$$
\mathbf{k}(t, \tau)=-\frac{1}{\tau} \int_{t-\tau}^{t} \mathbf{A}\left(t^{\prime}\right) d t^{\prime} .
$$

Although the function $\varphi(t, \tau)$ for arbitrary $t$ and $\tau$ has a quite complicated form (see Eqs. (9) and (3)), it reduces considerably at the saddle points $\tau=$ $\tau_{s}(t)$ owing to the simple form of its derivative with respect to $t$. Taking into account Eq. (13), this derivative may be written as follows:

$$
\mathrm{d} \varphi\left(t, \tau_{s}(t)\right) / \mathrm{d} t=-\left(\mathbf{k}\left(t, \tau_{s}(t)\right)+\mathbf{A}(t)\right)^{2} .
$$

As a result, the function $f_{\mathbf{p}}^{(1)}(t)$ in the saddle point approximation is represented as a sum of separate saddle point contributions and has the following form for a given $\tau_{s}$ :

$$
f_{\mathbf{p}}^{(1)}(t)=g_{s}(t) \mathrm{e}^{\mathrm{i}\left(E+u_{p}\right) t-\mathrm{i} \int^{t}\left(\mathbf{k}\left(t^{\prime}, \tau_{s}\left(t^{\prime}\right)\right)+\mathbf{A}\left(t^{\prime}\right)\right)^{2} \mathrm{~d} t^{\prime}}
$$

where $g_{s}(t)$ is a pre-exponential factor that depends smoothly on $t$.

Using Eq. (16), the equation for saddle points $t_{f}$ in the integral (5) for $\mathcal{A}_{n}^{(1)}$ is given by

$$
\left(\mathbf{k}\left(t_{f}, \tau_{s}\left(t_{f}\right)\right)+\mathbf{A}\left(t_{f}\right)\right)^{2}=\left(\mathbf{p}_{n}+\mathbf{A}\left(t_{f}\right)\right)^{2},
$$

where $\tau_{s}(t)$ satisfies Eq. (13). In contrast to the "direct scattering" process corresponding to the amplitude $\mathcal{A}_{n}^{(0)}$, relations (13) and (17) allow one to interpret the LAES process described by the amplitude $\mathcal{A}_{n}^{(1)}$ in terms of the two-step (rescattering) scenario: upon collision with the atom at the initial time $t_{i}=t_{f}-\tau_{s}$, the incoming electron changes its momentum from $\mathbf{p}$ to $\mathbf{k}$ (see 
Eq. (13)); then during the time $\tau_{s}$ it moves along a closed trajectory in the laser field, returning back to the origin at the moment $t_{f}=t_{i}+\tau_{s}$, whereupon it experiences a second collision that changes the "intermediate momentum" $\mathbf{k}$ to $\mathbf{p}_{n}$ (see Eq. (17)). Numerical values of $t_{f}$ and $\tau_{s}\left(t_{f}\right)$ are determined by Eqs. (13) and (17) and they may be either real or complex (corresponding to either classically allowed or forbidden motions of the electron in the intermediate state, after the first collision). As for the case of the $K$-plateau, the cutoff of the $R$-plateau corresponds to the minimum value of $p_{n}$ at which the roots $t_{f}$ and $\tau_{s}\left(t_{f}\right)$ acquire an imaginary part. For the case of linear polarization, the above considerations justify the use of one-dimensional classical equations to estimate the $K$ - and $R$-plateau cutoffs for forward and back scattering, which were given in Ref. [2] based upon physical arguments.

\subsection{Rescattering effects for the case of circular polarization}

For the general case of elliptic polarization, the analytical analysis of the rescattering plateau and of elliptic dichroism effects is cumbersome and will be published elsewhere. In what follows, we shall therefore restrict our consideration to the case of circular polarization, which is simpler for analytical analysis and for which the existence of high-energy plateau structures is somewhat unexpected. ${ }^{3}$ For $\xi= \pm 1$, Eqs. (13) and (17) simplify in such a way that the roots $t_{f}$ may be expressed explicitly in terms of $\tau_{s}$, resulting in a single equation that involves $\mathbf{p}_{n}, \mathbf{p}$ and $\tau_{s}$. (Physically, the dependence of $\mathbf{p}_{n}$ on only the return time $\tau_{s}$ in this case originates from the fact that the absolute value of the rotating vector $\mathbf{F}(t)$ is constant for $|\xi|=1$.) Consequently, the $R$-plateau cutoff for scattering in the plane of circular polarization is given by:

$$
p_{n, \max }^{(1)}(\theta)=\sqrt{u_{p}} \max \left[\sqrt{\sin ^{2} \psi(\varphi)+c(\varphi)}-\sin \psi(\varphi)\right],
$$

where

$$
\begin{aligned}
& \psi(\varphi)=\varphi+\xi \theta-\arcsin \frac{a^{2}-c(\varphi)}{2 a} \\
& a=p / \sqrt{u_{p}}, \quad c(\varphi)=\frac{4 \sin ^{2}(\varphi / 2)}{\varphi^{2}}-\frac{2 \sin \varphi}{\varphi}
\end{aligned}
$$

and the maximum in (18) is calculated over the set of return times $\tau_{s}$ ( $\varphi=$ $\left.\omega \tau_{s}\right)$. From Eq. (18) and the explicit form of $\psi(\varphi)$, one sees that the extent of

$\overline{3}$ Note that for this case the coefficients $f_{k}$ in Eq. (4) may be calculated analytically [2], so that the exact amplitude $\mathcal{A}_{n}$ is simplified and coincides with that obtained earlier in Ref. [9], in which, however, the high-energy plateau and circular dichroism effects were overlooked. 

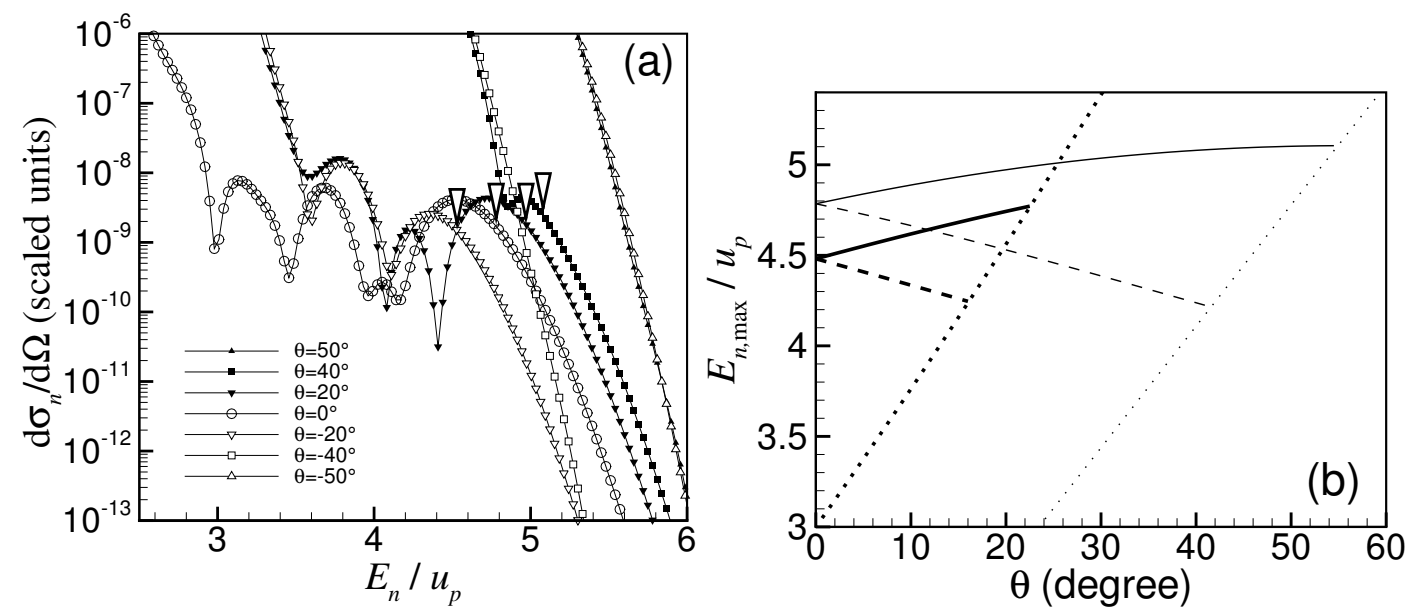

Fig. 2. (a) LAES spectra in the high energy ( $R$-plateau) region for different scattering angles $\theta$ in the polarization plane of a circularly polarized field with $\xi=+1$, $F=0.5$, and $\omega=0.155$, and for an incident electron energy $E=7.28=1.4 u_{p}$. Arrows mark the positions of the $R$-plateau cutoffs (according to Eq. (18)) for $\theta=-20^{\circ}, 0^{\circ}, 20^{\circ}$ and $40^{\circ}$ (from left to right). (b) Dependence of the $K$ - and $R$-plateau cutoffs on the scattering angle $\theta$ for $E=1.4 u_{p}$ (thin lines) and $E=3 u_{p}$ (thick lines), $F=0.5$ and $\omega=0.155$. Solid (dashed) lines: $R$-plateau cutoff positions for $\xi=+1(\xi=-1)$ according to Eq. (18); dotted lines: $K$-plateau cutoff positions (according to Eq. (11)).

the $R$-plateau depends on the sign of $\xi(\xi= \pm 1)$ only through the combination $\sin (\xi \theta)$. Expanding $p_{n, \max }^{(1)}(\theta)$ over $\theta$, we obtain for small-angle scattering:

$$
p_{n, \max }^{(1)}(\theta)=p_{n, \max }^{(1)}(0)\left[1-\frac{\xi \theta \cos \psi\left(\varphi_{m}\right)}{\sqrt{\sin ^{2} \psi\left(\varphi_{m}\right)+c\left(\varphi_{m}\right)}}\right],
$$

where $\varphi_{m}$ is the value of the parameter $\varphi$ in (18) at which $p_{n, \max }^{(1)}(0)$ is $\max$ imum. Analysis of the expression (18) shows that $\cos \psi\left(\varphi_{m}\right)<0$. Thus, according to (19), the cutoff of the $R$-plateau moves to higher energies with increasing $\theta$ for right-hand polarization $(\xi=+1)$ and to lower energies for left-hand polarization $(\xi=-1)$. This circular dichroism effect is illustrated in Fig. 2(a) (see also Fig. 1(b)), where the exact results for $R$-plateaus and the cutoff positions given by Eq. (18) are presented. We emphasize that dichroic effects disappear for "direct scattering" (in the $K$-plateau region), which is described well by the approximation (7) for the function $f_{\mathbf{p}}(t)$ in Eq. (5). Only a more precise account of the dynamics of the electron-atom interaction in the presence of a strong field can accurately predict dichroic effects in the high energy region beyond the cutoff of the $K$-plateau. As for the case of linear polarization $(\xi=0)[2]$, rescattering effects for the case of circular polarization are most important for small-angle scattering: for $\theta \gtrsim \theta_{c r}$, the $R$-plateau is masked by the more intense $K$-plateau (see Fig. $2(\mathrm{~b})$ ). Also, the critical angle $\theta_{c r}$ decreases with increasing $E$. 

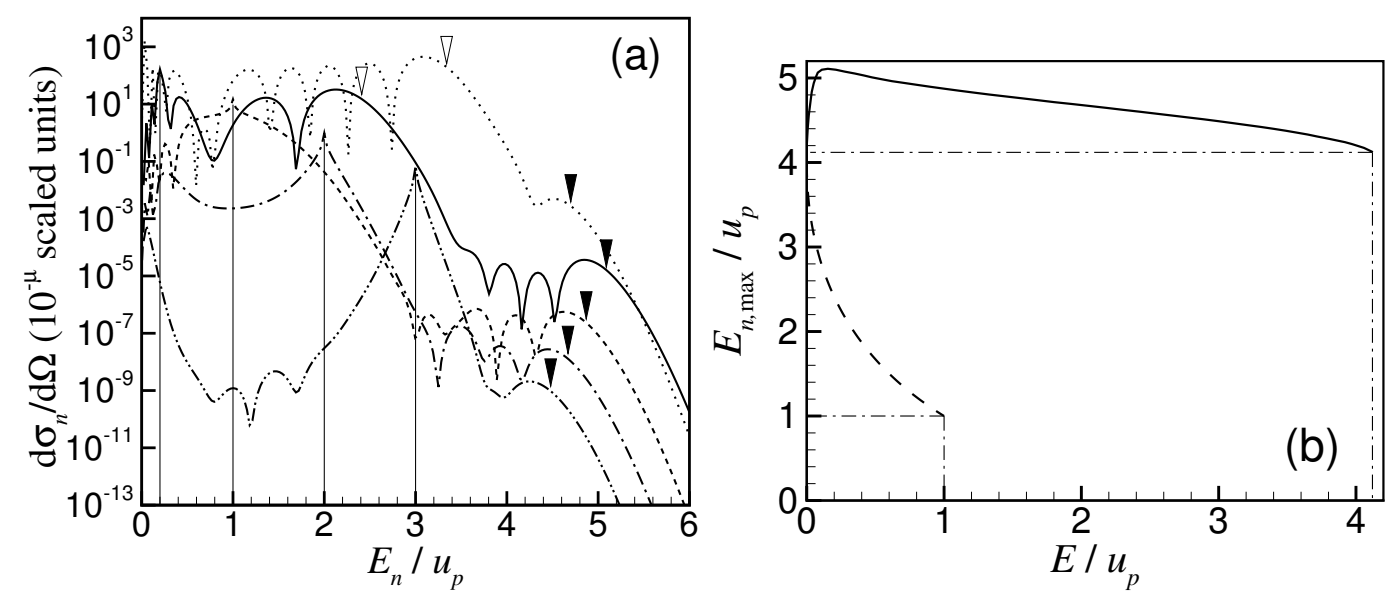

Fig. 3. (a) Energy distribution of electrons for forward scattering $(\theta=0)$ in the plane of circular polarization of a laser field with $F=0.5$ and $\omega=0.155$. Open and filled arrows mark the cutoffs of the $K$ - and $R$-plateaus according to (12) and (18) respectively. Vertical lines correspond to elastic scattering $\left(E_{n}=E\right)$. Each curve is multiplied by a factor $10^{\mu}$. Dotted line: $E=0.03 u_{p}, \mu=4$; solid line: $E=0.2 u_{p}$, $\mu=3$; dashed line: $E=u_{p}, \mu=2$; dot-dashed line: $E=2 u_{p}, \mu=1$; dot-dot-dashed line: $E=3 u_{p}, \mu=0$. (b) Dependence on $E$ of the cutoffs of the $K$ - (dashed line) and $R$ - (solid line) plateaus for forward scattering in the polarization plane of a laser field with $F=0.5, \xi=+1$, and $\omega=0.155$. Horizontal and vertical dot-dashed lines correspond to the coincidence of the cutoff positions with the elastic scattering peak at $E_{n, \max }=E$.

For forward scattering in the plane of circular polarization, in Fig. 3 we present the energy distribution of scattered electrons for different values of the incident electron energy $E$ (Fig. 3(a)) and the dependence of the cutoff positions on $E$ for both the $K$ - and $R$-plateaus (Fig. 3(b)). As shown analytically by analysis of Eqs. (13) and (17) for $\xi= \pm 1$ and $\theta=0$ and confirmed numerically by the results in Fig. 3(b), the extent of the $R$-plateau is $E_{n, \max }^{(1)}=4 u_{p}$ for $E \rightarrow 0$, which coincides with the global maximum for the extent of the $K$-plateau (12) for $E \rightarrow 0$. Although $E_{n, \max }^{(1)}$ increases rapidly with increasing $E$, reaching the global maximum, $E_{n, \text { max }}^{(1)} \approx 5.1 u_{p}$, at $E \approx 0.2 u_{p}$, for small energies $(E \rightarrow 0)$ the $R$-plateau is masked by the more intense $K$-plateau and, as shown by the numerical results, only becomes visible for $E \gtrsim 0.03 u_{p}$ (see the curve for $E=0.03 u_{p}$ in Fig. 3(a)). As for the case of the $K$-plateau (which exists for $E<u_{p}$ ), the $R$-plateau exists only for a limited interval of incoming electron energies $E$. A formal estimate for this interval follows from the condition that the "angle" $\psi\left(\varphi_{m}\right)$ must be real, i.e., $\left|a^{2}-c\left(\varphi_{m}\right)\right| \leq 2 a$, and results in the inequality $E<5.1 u_{p}$. However, due to the extra condition, $p<p_{n}$, the maximum in this inequality is reduced, so that the actual upper limit of energies $E$ for which the $R$-plateau exists decreases to $\sim 4.1 u_{p}$ (see Fig. 3(b)). Both parts of Fig. 3 show that the optimal interval of incident electron energies $E$ for observation of the $R$-plateau is $E \sim(0.2-2.5) u_{p}$, which depends on the laser intensity and frequency through the ponderomotive shift $u_{p}$. 
In conclusion, we have performed an accurate quantum analysis of plateau features in cross sections of electron-atom scattering assisted by a strong elliptically polarized laser field in the scattering length (ZRP model) approximation. We have derived the basic equations of the well-known rescattering scenario in strong laser-atom phenomena from our thoroughly quantum results for the scattering amplitude. We have also demonstrated the existence of high-energy (rescattering) plateaus for the case of circular laser polarization.

\section{Acknowledgements}

This work was supported in part by RFBR Grant 04-02-16350, by Grant E00-3.2-515 of the RF Ministry of Education, by the joint Grant VZ-010-0 of the CRDF and the RF Ministry of Education, and by Grant No. DEFG03-96ER14646 of the U.S. Department of Energy (AFS). AVF gratefully acknowledges the support of the Dynasty Foundation.

\section{References}

[1] P. Salières et al., Science 292 (2001) 902;

W. Becker, F. Grasbon, R. Kopold, D.B. Milošević, G.G. Paulus, H. Walther, Adv. At. Mol. Opt. Phys. 48 (2002) 35;

D.B. Milošević, F. Ehlotzky, Adv. At. Mol. Opt. Phys. 49 (2003) 373.

[2] N.L. Manakov, A.F. Starace, A.V. Flegel, M.V. Frolov, JETP Lett. 76 (2002) 258.

[3] M.Yu. Kuchiev, JETP Lett. 45 (1987) 404;

K.J. Schafer, B. Yang, L.F. DiMauro, K.C. Kulander, Phys. Rev. Lett. 70 (1993) 1599;

P.B. Corkum, Phys. Rev. Lett. 71 (1993) 1994.

[4] L.D. Landau, E.M. Lifshitz, Quantum Mechanics: Nonrelativistic Theory, 4th edn, Pergamon, Oxford, 1992, Section 133.

[5] M.V. Frolov, N.L. Manakov, E.A. Pronin, A.F. Starace, Phys. Rev. Lett. 91 (2003) 053003.

[6] N.L. Manakov, M.V. Frolov, B. Borca, A.F. Starace, J. Phys. B: At. Mol. Opt. Phys. 36 (2003) R49, Section 1.1.

[7] N.M. Kroll, K.M. Watson, Phys. Rev. A 8 (1973) 804.

[8] L.V. Keldysh, Sov. Phys. JETP 20 (1965) 1307.

[9] I.J. Berson, J. Phys. B: At. Mol. Phys. 8 (1975) 3078. 